\title{
Frontiers in immunology - grand challenges
}

\section{Kendall A. Smith*}

The Division of Immunology, Department of Medicine, Weill Medical College, Cornell University, New York, NY, USA

${ }^{*}$ Correspondence: kasmith@med.cornell.edu

As a science, immunology has now matured to the point where the tremendous complexities of the immune system are apparent for the first time. This new awareness of the daunting orchestration of antigen recognition, and the tempo, magnitude, duration, and character of the response, which is controlled by leukocytotrophic hormones, has become possible only within the past 30 years, as a result of the era of molecular immunology. In particular, we have recognized the complexities as a result of the capacity to delete or insert genes encoding important immunological molecules that have been discovered, so that we could observe their effects on the whole immune system in intact animals. Thus, the effects of the manipulation of individual genes often results in obvious immunological dysfunction that lead to lethal consequences. Other defects are more subtle, but often over time lead to equally devastating disruption of the immune system. Consequently, it is now evident that antigen recognition results in the activation of exquisite negative feedback loops in addition to the generation of marvelously effective immune responses. Thus, whether a detectable systemic immune response occurs or not is decided by the summation of these positive and negative molecular pathways.

It is also evident that genetic approaches can only go so far in providing information about the complex circuitry that results in immunological recognition and response. To progress in the future, it is clear that immunology will have to take chapters from the neuroscientists, and delve into the biophysics of intermolecular interactions. Thus, new ways of monitoring and measuring molecular concentrations and molecular changes, such as phosphorylations with their consequent conformational changes, will be necessary to discern how and why cellular behavior changes. In this regard, further reductionist approaches must be used at the single cell level, probing for molecular changes. Although Leonard Herzenberg introduced the flow cytometer to immunology almost 40 years ago (Julius et al., 1972), for the most part this methodology has only been used to illustrate the complexity of cell populations, even though it is possible to examine individual cells. The capacity to monitor and quantify intracellular and cell surface molecules with fluorescent monoclonal antibodies has been important in discerning how cell differentiation and signaling occurs (Jung et al., 1993; Perez and Nolan, 2002; Feinerman et al., 2008). However, the number of molecules that can be monitored simultaneously is limited by the number of fluorescent molecules. Now, the combination of flow cytometry and inductively coupled time-of-flight mass spectroscopy (Bandura et al., 2009) promises to be even more fruitful because it will be possible to determine the concentration and status of as many as 30 individual molecules within individual cells, and to relate this information to cellular behavior.

Although information as to the behavior of individual molecules in individual cells is necessary for a true reductionist approach to immunology, it is also important not to forget that the systemic immune response is determined by cell populations. Moreover, the various heterogeneous cells within the populations influence one another by expressing and metabolizing the many cell surface as well as secreted molecules. In this regard, it is no longer sufficient to view the immune system as passive, regulated totally from without upon introduction of antigenic molecules, a concept introduced by Burnet (1957). The cells of the system react to antigenic stimulation via expression of genes that encode leukocytotropic hormones, cell surface ligands, receptors, intracellular signaling molecules, and transcription factors, all of which determine the expression of genetic programs that ultimately determine cellular responses. Thus, the immune system is like other organ systems, being controlled by its own internally regulated hormone-like and neurotransmitter-like circuitry, and not solely by external antigens. The challenge that lies ahead is to unravel how this infinitely complex cellular and molecular circuitry eventually discriminates between self and non-self, and thereby leads to a recognizable and measurable immune response. It is clear that cells respond to ligand/receptor interactions at the cell surface in a quantal (all-or-none) manner, and somehow this digital information is transferred from the cell surface to the cytoplasm and to the nucleus, resulting in quantal gene expression (Smith, 2004, 2006, 2010). In this regard, systems biology approaches promise to provide much more data to decipher.

All of these issues become of paramount importance when considering disorders of the immune system that lead to immunological diseases. We have already made great progress in understanding the genetic and molecular defects that lead to primary as well as secondary immunodeficiencies, autoimmunity, allergies, and malignancies in the immune system. However, our abilities and capacities to therapeutically intervene in these disorders remains rudimentary. The dream to use monoclonal antibodies as therapeutics has finally been realized, and pharmaceutical companies are gradually transforming from being solely focused on small molecules to large molecules, particularly antibodies and cytokines targeting cells and extracellular molecules. However, in addition to these new therapeutics, we also need to generate more new and novel small molecular drugs that target specific intracellular molecules that we have uncovered as important for immunity.

One of the challenges ahead to more effectively treat immune system disorders is to combine the large molecular therapeutics that thus far have been utilized in the clinic only as monotherapies. The way forward has already been illuminated by the combination of anti-inflammatory agents with immunosuppressive drugs, which has created a revolution in the treatment of chronic inflammatory diseases such as rheumatoid arthritis (Maini et al., 1998; Lipsky et al., 2000). Other combinations such as antiinflammatory and immunostimulatory agents have yet to make it to the clinic, but 
they promise to provide for the ability to boost immune responses with fewer, or even completely absent inflammatory side effects. These ideas will undoubtedly form the basis of many new clinical trials that will lead to the acceptance, distribution and widespread use of immunotherapies. Who knows, they may even lead to a new kind of practitioner, one who understands the complexities of the immune system, can detect the abnormalities and can actually intervene to correct them!

One of the dreams that Pasteur promulgated was the hope that all infectious diseases could one day be prevented and treated simply by vaccination. Although Pasteur introduced the notion of attenuated live prophylactic vaccines in 1880 (Pasteur, 1880; Pasteur et al., 1881), and the idea of therapeutic vaccines for rabies in 1885 (Pasteur, 1885), we still cannot produce vaccines at will. Witness the saga of the difficulty of creating an HIV vaccine in our time (Smith, 2003). Even though we can determine the primary, secondary and tertiary structure of the site on the antigen molecule that binds to the receptor on the target cell surface, knowing this information still is not enough to develop a vaccine capable of eliciting virus neutralizing antibodies. Moreover, therapeutic vaccines for HIV and cancer are still in the realm of fantasy. Thus, another of our challenges is to understand the immune system well enough so that we can produce vaccines at will, which may well finally prevent and treat all infectious diseases, as well as cancer!

\section{REFERENCES}

Bandura, D. R., Baranov, V. I., Ornatsky, O. I., Antonov, A. Kinach, R., Lou, X., Pavlov, S., Vorobiev, S., Dick, J. E., and Tanner, S. D. (2009). Mass cytometry: technique for real time single cell multitarget immunoassay based on inductively coupled plasma time-of-flight mass spectrometry. Anal. Chem. 81, 6813-6822.

Burnet, F. M. (1957). A modification of Jerne's theory of antibody production using the concept of clonal selection. Aust. J. Sci. 20, 67-77.

Feinerman, O., Veiga, J., Dorfman, J., Germain, R., and Altan-Bonnet, G. (2008). Variability and robustness in $\mathrm{T}$ cell activation from regulated heterogeneity in protein levels. Science 321, 1081-1084.

Julius, M., Masuda, T., and Herzenberg, L. (1972). Demonstration that antigen-binding cells are precursors of antibody-producing cells after purification with a fluorescence-activated cell sorter. Proc. Natl. Acad. Sci. U.S.A. 69, 1934-1938.

Jung, T., Schauer, U., Heusser, C., Neuman, C., and Rieger, C. (1993). Detection of intracellular cytokines by flow cytometry. J. Immunol. Methods 159, 197-207.

Lipsky, P. E., van der Heijde, D. M., St Clair, E. W., Furst, D. E., Breedveld, F. C., Kalden, J. R., Smolen, J. S., Weisman, M., Emery, P., Feldmann, M., Harriman, G. R., and Maini, R. N. (2000). Infliximab and methotrexate in the treatment of rheumatoid arthritis. Anti-Tumor Necrosis Factor Trial in Rheumatoid Arthritis with Concomitant Therapy Study Group. N. Engl. J. Med. 343, 1594-1602.
Maini, R. N., Breedveld, F. C., Kalden, J. R., Smolen, J. S., Davis, D., Macfarlane, J. D., Antoni, C., Leeb, B., Elliott, M. J., Woody, J. N., Schaible, T. F., and Feldmann, M. (1998). Therapeutic efficacy of multiple intravenous infusions of anti-tumor necrosis factor alpha monoclonal antibody combined with low-dose weekly methotrexate in rheumatoid arthritis. Arthritis Rheum. 41, 1552-1563.

Pasteur, L. (1880). Sur les maladies virulentes, et en particulier sur la maladie appelee vulgairement cholera des poules. C. R. Hebd. Seances Acad. Sci. 90, 249-248.

Pasteur, L. (1885). Methode pour prevenir la rage apres morsure. C. R. Acad. Sci. Seances 101, 765-774.

Pasteur, L., Chamberland, C., and Roux, E. (1881). De l'attenuation des virus et de leur retore a la virulence. C. R. Seances Acad. Sci. 92, 430-435.

Perez, O., and Nolan, G. (2002). Simultaneous measurement of multiple kinase states using polychromatic flow cytometry. Nat. Biotechnol. 20, 155-162.

Smith, K. (2003). The HIV vaccine saga. Med. Immunol. 2 , 1.

Smith, K. (2004). The quantal theory of how the immune system discriminates between "self and non-self". Med. Immunol. 3, 3.

Smith, K. (2006). The quantal theory of immunity. Cell Res. 16, 11-19.

Smith, K. A. (2010). The Quantal Theory of Immunity. Singapore: World Scientific Publishing Co. Pte. Ltd.

Received: 16 July 2010; accepted: 16 July 2010; published online: 19 October 2010.

Citation: Smith KA (2010) Frontiers in immunology grand challenges. Front. Immun. 1:1. doi: 10.3389/ fimmu.2010.00001

Copyright $(2010$ Smith. This is an open-access article subject to an exclusive license agreement between the authors and the Frontiers Research Foundation, which permits unrestricted use, distribution, and reproduction in any medium, provided the original authors and source are credited. 